\title{
Inhalt
}

\section{Abkürzungsverzeichnis - XXI}

\section{Kapitel 1: Einführung - 1}

$\S 1$ Rechtspolitische Ausgangslage - 1

$\S 2$ Gegenstand und Gang der Untersuchung - 4

I. Ein dreigeteilter Untersuchungsgegenstand $-\mathbf{4}$

II. Methodisches Vorgehen und Verortung des Untersuchungsgegenstandes -5

1. Rechtsdogmatik -5

2. Rechtssoziologie und Rechtspolitik - 6

III. Gang der Untersuchung - 6

Kapitel 2: Tarifautonomie - Bedeutungskraft und Herausforderungen - 9

§ 3 Tarifautonomie als Bestandteil der Arbeitsrechtsordnung - 9

I. Begriff und Funktionen der Tarifautonomie -9

1. Schutz- und Verteilungsfunktion - $\mathbf{1 1}$

2. Ordnungs- und Befriedungsfunktion -12

3. Faktische Kartellwirkung des Flächentarifvertrags - 13

II. Tarifautonomie als Verfassungswert -14

1. Tarifautonomie als Ausfluss kollektiver Betätigungsfreiheit - $\mathbf{1 4}$

2. Tarifautonomie zwischen Ausgestaltung und Eingriff - 14

3. Tarifautonomie und Arbeitskampffreiheit - 15

$\S 4$ Die Entwicklung der Tarifautonomie - eine historische Skizzierung - 16

I. Die Entwicklungslinien der Tarifautonomie bis $1871-16$

1. Der Beginn kapitalistischer Produktionsweise als Ausgangspunkt -17

2. Preußische Koalitionsverbote $-\mathbf{1 7}$

II. Tarifautonomie im Deutschen Kaiserreich - 19

1. Bismarcksche Sozialistengesetzgebung - 19

2. Tarifautonomie im Aufschwung - 20

III. Tarifautonomie in der Weimarer Republik -20

1. Hugo Sinzheimer als Architekt des Tarifvertragssystems - 21

2. Die erste „Blütezeit“ der deutschen Tarifautonomie - 22

a) Tarifvertragsverordnung - $\mathbf{2 2}$

b) Verfassungsrechtliche Verankerung - 23

IV. Tarifautonomie in der Zeit des deutschen Nationalsozialismus - 23 
V. Tarifautonomie im Nachkriegsdeutschland (seit 1945) - 24

1. Der Neuaufbau der Gewerkschaften - $\mathbf{2 4}$

2. Erneute Kodifizierungen $-\mathbf{2 5}$

3. Tarifpolitische Erfolge $-\mathbf{2 6}$

$\S 5$ Tarifautonomie heute: veränderte Rahmenbedingungen — 27

I. Mitgliederschwund bei den Gewerkschaften - $\mathbf{2 7}$

1. Mitgliederentwicklung des Deutschen Gewerkschaftsbundes $-\mathbf{2 8}$

2. Mitgliederzahlen bei sonstigen Gewerkschaften: DBB und Marburger Bund -29

3. Mögliche Ursache: fehlende ökonomische Anreize? - $\mathbf{3 0}$

4. Sinkende Tarifbindung der Arbeitnehmer — 31

II. Arbeitsvertragliche Bezugnahme auf Tarifverträge - 32

1. Motivation: Ordnungswirkung und Schwächung der Gewerkschaften? - 33

2. Empirie und Relevanz für die Untersuchung - 34

III. Differenzierungsklauseln im Tarifvertrag - 34

1. Motivation: Lösung der Trittbrettfahrerproblematik? - 35

2. Empirie und Relevanz für die Untersuchung - 35

IV. Mitgliederschwund bei den Arbeitgeberverbänden? - 36

1. Anhaltspunkt: Mitgliederentwicklung des Arbeitgeberverbands Gesamtmetall — 37

2. Mögliche Ursachen: Flexibilisierung und Tarifflucht - 37

3. Sinkende Tarifbindung der Arbeitgeber - 38

V. OT-Mitgliedschaften -39

1. Motivation - $\mathbf{4 0}$

2. Empirie und Relevanz für die Untersuchung - $\mathbf{4 0}$

VI. Die zunehmende Bildung von Spartengewerkschaften - 41

VII. Die Reaktionen des Bundesgesetzgebers — 43

1. Das Tarifautonomiestärkungsgesetz — 43

a) Erleichterte Möglichkeit der Allgemeinverbindlicherklärung - 43

b) Erweiterte Gestaltungsfreiheit für Rechtsverordnungen nach dem AEntG — $\mathbf{4 5}$

c) Flächendeckender gesetzlicher Mindestlohn - 47

2. Das Tarifeinheitsgesetz $-\mathbf{4 8}$

a) Die Einführung des § 4 a TVG -48

b) Motivation: Sicherung der Funktionsfähigkeit der Tarifautonomie - $\mathbf{5 0}$

c) Die Entscheidung des BVerfG vom 11. Juli $2017-50$ 
VIII. Quintessenz: Tarifbindung im Fadenkreuz - 52

§ 6 Zusammenfassung von Kapitel 2 und Schlussfolgerungen - 53

\section{Kapitel 3: Faktoren einer funktionsfähigen Tarifautonomie - $\mathbf{5 5}$}

$\S 7$ Weichenstellung: der Ursprung der normativen Wirkung des Tarifvertrags -56

I. Staatlich-delegatorische Ansätze - $\mathbf{5 7}$

1. „Klassische“ Delegationstheorie - $\mathbf{5 7}$

2. Integrationstheorie - $\mathbf{5 8}$

II. Autonomietheoretische Ansätze - $\mathbf{5 9}$

1. Die vorstaatliche Autonomietheorie - $\mathbf{5 9}$

2. Legitimations- und Anerkennungstheorie - $\mathbf{5 9}$

3. Rechtsgeschäftliche Ansätze - 61

III. Synergetische Ansätze - 63

1. Die Sanktionstheorie - 63

2. Der „dynamische“ Kombinationsansatz — 64

IV. Die normativen Wirkung des Tarifvertrags im Spiegel der Rechtsprechung - 64

1. Die Rechtsprechung des BVerfG -64

2. Die Rechtsprechung des BAG -66

a) Delegationstheorie -66

b) Paradigmenwechsel: die Akzeptanz der Legitimationstheorie -66

V. Stellungnahme -67

1. Erklärungsdefizite der rechtsgeschäftlichen Ansätze — 68

2. Möglichkeit privater Normsetzung -69

3. Erklärungsdefizite der staatlich-delegatorischen Ansätze - $\mathbf{7 1}$

4. Erklärungsdefizite der autonomietheoretischen Ansätze $-\mathbf{7 2}$

5. Erklärungsdefizite der synergetischen Ansätze - 73

6. Ein Plädoyer für die Legitimationstheorie - Tarifautonomie als kollektiv ausgeübte Privatautonomie -74

VI. Folgen für die Grundausrichtung des Tarifsystems - 77

1. Auf Mitgliedschaft basierende Tarifautonomie - $\mathbf{7 8}$

2. Begrenzte Ordnungsfunktion der Tarifautonomie - $\mathbf{7 9}$

3. Keine Legitimation zur Regelung von AußenseiterArbeitsverhältnissen $-\mathbf{8 0}$

4. Privatautonomer Koalitionspluralismus $-\mathbf{8 0}$

5. Mittelbare Grundrechtsbindung der Tarifvertragsparteien — $\mathbf{8 1}$ 
§ 8 Ableitung der Faktoren einer funktionsfähigen Tarifautonomie - $\mathbf{8 1}$

I. Faktor 1: die Verbandsmitgliedschaft des Arbeitnehmers - $\mathbf{8 2}$

1. Der privatautonome Geltungsgrund des Tarifvertrags - $\mathbf{8 2}$

2. Verfassungsrechtliche Implikationen - $\mathbf{8 3}$

3. Einfachgesetzlicher Ausdruck gem. §§ 4 Abs. 1, 3 Abs. 1 TVG -84

4. Das Erfordernis der Tariffähigkeit - $\mathbf{8 5}$

a) Die Durchsetzungskraft einer Arbeitnehmerkoalition - $\mathbf{8 6}$

b) Stellenwert der Gewerkschaftsmitgliedschaft im Spiegel der Rechtsprechung — $\mathbf{8 7}$

c) Erkenntnisse und Rückschlüsse für eine funktionsfähige Tarifautonomie - $\mathbf{8 9}$

d) Tariffähigkeit und Tarifautonomie - ein Widerspruch? — 89

5. Die Richtigkeitsvermutung des Tarifvertrags - $\mathbf{9 0}$

II. Faktor 2: die Verbandsmitgliedschaft des Arbeitgebers — 92

1. Rechtsdogmatische Gemeinsamkeiten und Unterschiede zur Mitgliedschaft der Arbeitnehmer — 92

2. Einwand: der einzelne Arbeitgeber als Tarifakteur i.S.d. § 2 Abs. 1 TVG 93

a) Die Tariffähigkeit des einzelnen Arbeitgebers — 93

b) Der idealtypische Vorrang des Flächentarifvertrags - 94

III. Faktor 3: Interessenpluralität durch repräsentative Tarifverträge -96

1. Der privatautonome Geltungsgrund des Tarifvertrags - $\mathbf{9 7}$

2. Ausgangspunkt: Koalitionspluralismus aus Art. 9 Abs. 3 Satz 1 GG 98

3. Vom Koalitionspluralismus zum Gewerkschaftspluralismus - 99

4. Vom Gewerkschaftspluralismus zur Tarifpluralität - $\mathbf{1 0 0}$

IV. Faktor 4: der Schutz des tarifautonomen Regelungsbereichs - 101

1. Der privatautonome Geltungsgrund des Tarifvertrags - $\mathbf{1 0 2}$

2. Verfassungsrechtliche Verankerung - $\mathbf{1 0 2}$

3. Schutz des tarifautonomen Regelungsbereichs nach $\S 77$ Abs. 3 BetrVG 103

V. Faktor 5: Vorrang der mitgliedschaftlich legitimierten Tarifnormgeltung - 104

1. Der privatautonome Geltungsgrund des Tarifvertrags - $\mathbf{1 0 5}$

2. Außenseiterwirkung von Tarifnormen und gegenläufige Verfassungsprinzipien 106

a) Demokratieprinzip — 106

b) Arbeitsvertragsfreiheit -107 
c) Koalitionsfreiheit und negative Tarifvertragsfreiheit 108

3. Einfachgesetzliche Verankerung des Regel-AusnahmeVerhältnisses — 111
a) Regelfall: Tarifnormgeltung gem. §§ 4 Abs. 1, 3 Abs. 1 TVG — 111
b) Ausnahmetatbestände - 111
c) Systematische Erwägungen — 114

VI. Das Verhältnis der Faktoren zueinander — 114

1. Zusammenhänge - $\mathbf{1 1 4}$

2. Hierarchisierung - $\mathbf{1 1 6}$

§ 9 Zusammenfassung und Zwischenergebnisse von Kapitel 3-116

\section{Kapitel 4: Tarifpolitik und Tarifautonomiestärkungsgesetz — 119}

$\S 10$ Differenzierung: empirischer und rechtlicher Zustand der Tarifautonomie - $\mathbf{1 2 0}$

I. „Empirischer“ Zustand der Tarifautonomie — 120

II. „Rechtlicher“ Zustand der Tarifautonomie - 121

$\S 11$ Stabilisierung durch Differenzierungsklauseln und OTMitgliedschaften? - 122

I. Einordnung und Bewertung der Differenzierungsklausel -123

1. Erscheinungsformen der Differenzierungsklausel - $\mathbf{1 2 4}$
a) Die einfache Differenzierungsklausel — 124
b) Die Spannenklausel - $\mathbf{1 2 5}$
c) Die Tarifausschlussklausel $\mathbf{1 2 5}$
d) Die Stichtagsklausel $\mathbf{1 2 6}$

2. Differenzierungsklauseln im System der Tarifautonomie perspektivische Betrachtungsweisen - 127

3. Differenzierungsklauseln im Spiegel der Rechtsprechung des BAG -128
a) Die einfache Differenzierungsklausel 129
b) Die Spannenklausel 132
c) Die Tarifausschlussklausel 133
d) Die Stichtagsklausel 133

4. Stellungnahme -134
a) Zum Prüfungsmaßstab der Koalitionsfreiheit — 136
b) Zum Prüfungsmaßstab einer negativen Tarifvertragsfreiheit 144
c) Zum Prüfungsmaßstab der Arbeitsvertragsfreiheit — 145
d) Zum Prüfungsmaßstab des allgemeinen Gleichheitssatzes — 147 
e) Rechtspolitische Folgerungen - 148

II. Einordnung und Bewertung der OT-Mitgliedschaft -149

1. Rechtliche Konstruktion und Abgrenzung - $\mathbf{1 5 0}$

2. Die OT-Mitgliedschaft im System der Tarifautonomie - $\mathbf{1 5 1}$

3. Die OT-Mitgliedschaft im Spiegel der Rechtsprechung des $B A G-152$

a) Ausgangspunkt: Die Entscheidung des BAG vom 23. Februar $2005-152$

b) Bestätigung und Fortführung: Die Entscheidung des BAG vom 18. Juli $2006-153$

c) „Blitzwechsel“-Rechtsprechung: Die Entscheidung des BAG vom 4. Juni $2008-154$

d) Bestätigung und weitere Ausdifferenzierung der Blitzwechselrechtsprechung - 155

e) Quintessenz - 156

4. Stellungnahme - $\mathbf{1 5 7}$

a) Einfachrechtliche Grenzen - 157

b) Das Gebot der Verhandlungsparität als verfassungsrechtliche Grenze - 163

c) Zur Rechtsfolge eines unwirksamen Wechsels in die OTMitgliedschaft - 168

d) Rechtspolitische Bewertung: Dysfunktionalität der OTMitgliedschaft -173

III. Stärkung der Tarifautonomie als Aufgabe des Gesetzgebers - 175

$\S 12$ Einordnung und Bewertung des Tarifautonomiestärkungsgesetzes — 176

I. Die erleichterte Möglichkeit der

Allgemeinverbindlicherklärung — $\mathbf{1 7 7}$

1. Funktionen der Allgemeinverbindlicherklärung - 177

a) Schutzfunktion: Gewährleistung angemessener Mindestarbeitsbedingungen -178

b) Finanzierungsfunktion: Sicherung gemeinsamer Einrichtungen -178

c) Kartell- und Wettbewerbsfunktion? - 179

d) Stärkung der Tarifautonomie? - $\mathbf{1 8 0}$

2. Dogmatische Probleme der Neuregelung von $\S 5$ TVG -182

a) Zum Begriff des öffentlichen Interesses in $\S 5$ Abs. 1 TVG - 182

b) Zur Allgemeinverbindlicherklärung eines Tarifvertrags über gemeinsame Einrichtungen - 189

c) Schlussfolgerung - 193 
3. Berücksichtigung verfassungsrechtlicher Maßstäbe - 194

a) Tarifnormerstreckung im Spiegel der Rechtsprechung des BVerfG 194

b) Verschärfter Konflikt mit verfassungsrechtlichen Wertungen? - 202

c) Rechtfertigung und verfassungskonforme Auslegung — 211

4. Rechtspolitische Bewertung: Schwächung der Tarifautonomie - $\mathbf{2 2 5}$

II. Die Einführung eines flächendeckenden gesetzlichen Mindestlohns $\mathbf{2 2 6}$

1. Zur tarifverdrängenden Wirkung des Mindestlohns, § 3 Satz 1 MiLoG 227
a) Verfassungsrechtlicher Eingriff in die Tarifautonomie? -227
b) Rechtfertigung? - $\mathbf{2 2 9}$
c) Rechtspolitische Kritik: Begrenzung des tarifautonomen Regelungsbereichs -234

2. Zum Anpassungsverfahren, $\S \S 4 \mathrm{ff}$. MiLoG — 234

a) Dogmatische Skizze - 234

b) Vereinbarkeit mit dem Demokratieprinzip? — 236

c) Vereinbarkeit mit der Wesentlichkeitstheorie? - 241

d) Alternativvorschlag: der Gesetzesentwurf des Bundesrats -242

III. Die erweiterte Tarifnormerstreckung nach den Regeln des AEntG 243

1. Zur Genese des AEntG — 243

2. Dogmatische Skizzierung des Rechtsverordnungsverfahrens für die Tarifnormerstreckung in anderen Branchen, §§7a, 4 Abs. 2 AEntG 245

3. Verfassungsrechtliche Kritikpunkte - $\mathbf{2 4 6}$

a) Verschärfter Konflikt mit dem Demokratieprinzip? - 246

b) Kompensation durch zusätzliche Voraussetzungen? — 247

4. Rechtspolitische Kritik — 249

$\S 13$ Zusammenfassung und Zwischenergebnisse von Kapitel $4-250$

Kapitel 5: Ansätze und Vorschläge zur Stärkung der Tarifautonomie — 253 $\S 14$ Gesetzlicher Spanneneffekt — 255

I. Vorschlag: Gesetzliche Absicherung des „Spanneneffekts“ — 256

II. Anforderungen an die gesetzliche Formulierung - 258 
III. Kommentierung -259

1. Abs. 1: Zulässigkeit einfacher Differenzierungsklauseln - 259

2. Abs. 2: Gesetzliche Absicherung des Spanneneffekts - 260

3. Abs. 3: Verantwortung der Gewerkschaften zur praktischen Umsetzung — 261

IV. Verfassungsrechtliche Gesichtspunkte - 261

1. Die negative Koalitionsfreiheit als Prüfungsmaßstab - 261

2. Die Arbeitsvertragsfreiheit als Prüfungsmaßstab - 263

a) „Gemeinwohlbelang“ als maßgebliche Rechtfertigungshürde -264

b) Sicherung der Funktionsfähigkeit der Tarifautonomie - 265

c) Einschätzungsprärogative des Gesetzgebers — 268

d) Keine Überschreitung der Tarifmacht — 268

V. Praktische Abwicklung - 269

1. Die Frage nach der Gewerkschaftszugehörigkeit als rechtspraktische Hürde -269

2. Durchführungsmöglichkeit -271

VI. Ergebnis - $\mathbf{2 7 2}$

$\S 15$ Das skandinavische Modell der Sozialpartnerschaft — 273

I. Rechtstatsachen: Gewerkschaftlicher Organisationsgrad im europäischen Vergleich — 273

II. Das skandinavische Modell der Sozialpartnerschaft - $\mathbf{2 7 5}$

1. Regelung der Arbeits- und Wirtschaftsbedingungen - $\mathbf{2 7 5}$

2. Einbindung in die Systeme der sozialen Sicherheit $-\mathbf{2 7 7}$

3. Beteiligung an wirtschaftspolitischen Entscheidungen $-\mathbf{2 7 8}$

III. Zentrale Erkenntnisse - $\mathbf{2 7 8}$

IV. Das Genter-System als Möglichkeit zur Stärkung der Tarifautonomie? - $\mathbf{2 7 9}$

1. Das „historische“ Genter-System - $\mathbf{2 8 0}$

a) Hinweise zur gesetzgebungstechnischen Implementierung - $\mathbf{2 8 2}$

b) Verfassungsmäßigkeit $-\mathbf{2 8 3}$

c) Rechtspolitische Kritik — $\mathbf{2 8 8}$

d) Ergebnis - $\mathbf{2 8 8}$

2. Spielarten des Genter-Systems — 289

a) Spielart 1: Differenzierung hinsichtlich der Beitragshöhe - $\mathbf{2 8 9}$

b) Spielart 2: Ohne Differenzierung hinsichtlich der Beitragshöhe - 292

V. Ergebnis - 294 
$\S 16$ Differenzierung auf Arbeitgeberseite und tarifdispositives Gesetzesrecht — 294

I. Mögliche Anknüpfungspunkte für eine Differenzierung — 296

1. Steuer- und Beitragslast -296

2. Tarifdispositives Gesetzesrecht — 297

II. Fokussierung: Tarifdispositives Gesetzesrecht als Maßnahme zur Stärkung der Tarifautonomie? - 298

1. Wirkungsweise: Licht und Schatten -298

a) Relativierung: Kompensationsgeschäfte und Richtigkeitsvermutung - $\mathbf{3 0 0}$

b) Stärkung der Tarifautonomie? - $\mathbf{3 0 2}$

2. Das „Trittbrettfahrerproblem“ auf Arbeitgeberseite — 303

3. Lösungskonzepte - 305

a) Bisherige Vorschläge: Verknüpfung der Bezugnahme mit Tarifbindung — 305

b) Eigener Vorschlag: Verknüpfung der Bezugnahme mit Verbandsmitgliedschaft $-\mathbf{3 1 0}$

4. Verfassungsrechtliche Grenzen - $\mathbf{3 1 1}$

a) Negative Koalitionsfreiheit - $\mathbf{3 1 2}$

b) Berufs- bzw. Unternehmensfreiheit und allgemeiner Gleichheitssatz 316

III. Ergebnis - 318

$\S 17$ Zeitliche Begrenzung von Nachbindung und Nachwirkung? — 318

I. Ewigkeitsbindung an Tarifverträge? - $\mathbf{3 1 9}$

1. Phase 1: Die Nachbindung an Tarifverträge, $\S 3$ Abs. 3 TVG 320

a) Reichweite und Grenzen der Nachbindung - 320

b) Keine zeitliche Begrenzung de lege lata - 322

2. Phase 2: Die Nachwirkung von Tarifverträgen, $\S 4$ Abs. 5 TVG 323

a) „Ablauf des Tarifvertrags“ 324

b) „Andere Abmachung“ _ 324

3. Bewertung — 326

a) Zur Nachwirkung gem. § 4 Abs. 5 TVG -327

b) Zur Nachbindung gem. § 3 Abs. 3 TVG -328

II. Vorschlag: Gesetzliche Begrenzung der Nachbindungsdauer — 329

1. Kriterien der Begrenzungsdauer - $\mathbf{3 3 0}$

a) Ausgangspunkt: regelmäßige Dauer von

Tarifverträgen $-\mathbf{3} 30$ 
$\mathbf{X X}$ Inhalt

b) Die ratio legis des $\S 3$ Abs. 3 TVG als zeitliche Untergrenze - 331

c) §613a Abs. 1 Satz 2 BGB als Vergleichsgrundlage? — 332

d) Schlussfolgerung - 334

2. Fristbeginn und Jahresdauer als Maximalgrenze - 334

3. Formulierungsvorschlag $-\mathbf{3 3 5}$

III. Ergebnis - 335

Kapitel 6: Zusammenfassende Thesen und Empfehlungen — 337

Literaturverzeichnis - 343 